\title{
HUMANIZACIÓN \\ DE LA EDUCACIÓN
}

Carmen Rosa Villarán

Docente de la Facultad de Teología Pontificia y Civil de la Universidad Católica de Lima (Perú)

\subsection{La admiración sobre el ser humano y el descubrimiento de una naturaleza personal}

Se podría afirmar, desde el sentido común: cuán necesario es en nuestros días admirarse, reflexionar y examinar la conducta característica de los miembros del género humano en todos los tiempos. Siendo capaces de considerar la presencia de un notable afán de felicidad junto a un continuo deseo de ser mejores. Todo lo cual, en las últimas décadas como en otras épocas, es susceptible de ser apreciado a través de las obras humanas, las mismas que, paradójicamente según su intención, ejecución y producción, en la práctica, muchas veces se han tornado contrarias al beneficio de los hombres. De tal modo que es preponderante para el género humano impedir desaciertos teóricos, prácticos y productivos en el comportamiento de los hombres; precisamente, por las consecuencias adversas frente al mejoramiento y felicidad de cada ser humano que devendrá en el de toda la humanidad.

En fin, no hay duda de que el hombre tiene poder para tergiversar su realidad personal, a la vez que es capaz de encaminarla y realizarla convenientemente; lo 
que contiene un peligro, pero también, una expectativa que puede redundar en esperanza. Por lo mismo y realmente para acertar con la correspondiente conducta humana, es primordial la profundización en la naturaleza humana, en su índole radical de persona, en razón de sus fines intermedios y del fin último abierto a la perfección y así, a la felicidad.

Asimismo, al compás de la historia del mundo en general y de la humanidad en particular, el hombre ha reconocido su sitial preponderante y ha ido comprendiendo también que cazar, pescar, construir casas, etc., tiene su dificultad; pero más difícil es formar la propia personalidad y cooperar en la de los demás; que es, simultáneamente, lo más nuclear de su existencia. No hay duda, "que la persona significa aquello que es perfectísimo en toda la naturaleza"1; puesto que "la manera de existir propia de la persona, es la más excelente de todas". ${ }^{2}$

Sin duda han habido épocas románticas en que se ha puesto énfasis en la naturaleza, la patria..., pero de un modo u otro nunca ha dejado de estar patente, aunque incluso en nuestros días parezca olvidada la dimensión trascendental, inscrita en lo personal y apreciable en aquella postura agustiniana: "Quiero conocer a Dios y al alma... No sé cómo debe manifestárseme Dios para que diga: ya es suficiente, porque no creo que conozca ninguna cosa como deseo conocerlo a Él..." En esta línea San Agustín afirmaba: "Hombres son y no los amo por su naturaleza animal, sino humana; esto es, porque tienen almas racionales, que yo aprecio hasta en los ladrones".3

Precisamente, ocuparse del hombre como persona es separarlo de la condición de las cosas, reconocerle una realidad superior en razón de su alma que representa para los seres animales "primordialmente y de ma- 
nera especialísima el elemento motor". ${ }^{4}$ Así, "todos aquellos que se fijaron en el hecho de que el ser animado se mueve, supusieron que el alma es el motor por excelencia". 5 Por tanto, "si cabe enunciar algo en general acerca de toda clase de alma, habría que decir que es la entelequia primera de un cuerpo natural organizado"6. Queda así entendida el alma como causa, principio y también fin; "y este fin constituye su perfección".7 Así, en cuanto que su alma tiene una determinada naturaleza, su perfección consistirá en realizarla. En consecuencia, el caso del hombre es único en cuanto que su alma es susceptible, según su naturaleza intelectual, de dar cuenta de sí misma y también dirigirse por sí misma.

Así pues, la calidad racional del alma del ser humano, es la base para hablar del hombre como persona y reconocer en él una naturaleza muy superior a todas las criaturas. La misma racionalidad del alma humana permite la exclusividad de la voluntad para elegir, decidir, etc. Asimismo, la racionalidad pone de relieve y permite la libertad. Sin duda, el hombre nota que aspira a saciar su "libre" anhelo de felicidad; y persigue por tanto, un buen desenlace de su íntima condición perfectiva. En este sentido, es necesario que conozca acabadamente "qué y quién es". Considérese por ello, este requerimiento a la luz de una profunda indagación agustiniana que tiene mucho de filosofía cristiana: "Entonces me dirigí a mí mismo y me dije: ‘'tú, quién eres?', y respondí: “un hombre”. He aquí, pues, que tengo en mí prestos un cuerpo y un alma; el uno interior, el otro exterior. ¿Por cuál de éstos es por donde debí yo buscar a mi Dios, a quien ya había buscado por los cuerpos desde la tierra al cielo, hasta donde pude enviar los mensajeros rayos de mis ojos? Mejor, sin duda, es el elemento interior, porque a él es a quien comunican sus noticias todos los mensajeros cor- 
porales, como a presidente y juez, de las respuestas del cielo, de la tierra, y de todas las cosas que en ellos se encierran, cuando dicen: "No somos Dios" y "Él nos ha hecho". El hombre interior es quien conoce estas cosas por ministerio del exterior; yo interior conozco estas cosas; yo, yoalma, por medio del sentido de mi cuerpo. Interrogué finalmente, a la mole del mundo acerca de mi Dios, y ella me respondió: "No lo soy yo, simple hechura suya... Dios es para ti la vida de tu vida".

Así, indagar en el ser del hombre, en cuanto animado racionalmente según su propia libertad (decisión), y adentrarse en su alma como núcleo de dicha animación, resulta un camino serio para la posibilidad científica de conocerlo y de abordarlo en toda su integridad, y poder denominarlo en último término; como ya se hizo más adelante como persona, lo que en sentido estricto, apunta a la más alta dignidad en orden a la posesión del alma más perfecta entre los seres corpóreos. La condición humana queda de relieve como portadora conjuntamente y de modo preferente desde su alma, tanto de una racionalidad como de una voluntariedad. Cabe así precisar que, desde la investigación, en la misma animación humana: todo hombre es persona por su racionalidad y voluntad, facultades que se manifiestan en estado de libertad, y "a su vez, la libertad no es posible sin el entendimiento".

En tal sentido, "la libertad, que capacita al hombre para obrar por deber y no solamente por instinto, es también lo que le hace ser persona y no un simple animal. Llamamos, en efecto, personas, a diferencia de los animales y también de las cosas, a los seres que tienen libertad. Todo hombre es persona por tener una cierta libertad, aun en las peores circunstancias. Y el hecho de que esta libertad pueda ser íntimamente trasgredida o incluso externamente violentada no se elimina su naturaleza ni la dig- 
nidad de la "persona humana", que, aunque está por debajo de la de Dios, es evidentemente superior a la naturaleza de los animales y las cosas". 10

\subsection{La comprensión y mejora educativa del ser humano a través de sus acciones}

En razón del viejo adagio de raigambre metafísica: "El modo de obrar sigue al modo de ser", tenemos que dentro de una clara perspectiva antropológica, se puede constatar que lo objetivo y lo subjetivo, lo absoluto y relativo, lo trascendente y lo inmanente, lo permanente y lo contingente, se hace más nítido en la experiencia de la acción humana. "La acción constituye el momento específico por medio del cual se revela la persona. La acción nos ofrece el mejor acceso para penetrar en la esencia intrínseca de la persona y nos permite conseguir el mayor grado posible de conocimiento de la persona. Experimentamos al hombre en cuanto persona y estamos convencidos de ello porque realizamos acciones...."11

Ahora bien, en razón de las mismas acciones humanas, que pueden notarse resueltas al interior y/o exterior del mismo ser del hombre, téngase en cuenta que en el discernimiento (acción inmanente), se procede "de las cosas que son para nosotros más conocidas y claras a aquellas otras que son más claras y más cognoscibles en sí”. ${ }^{12}$ Como dice en otra parte el Filósofo, "sin duda se ha de empezar por las cosas más fáciles de conocer; pero éstas lo son en dos sentidos: uno para nosotros, las otras en absoluto. Debemos, pues, acaso empezar por las más fáciles de conocer para nosotros". ${ }^{13}$ Entiéndase, por tanto, cómo mediante la acción, la persona para sí misma y para los demás aparece y se constituye como tal; además, dicha acción puede resolverse en el interior $y / o$ trascender al exterior. 
En cuanto a la acción educativa, la primera comprensión elemental nos remite al ser humano como sujeto exclusivo y de modo más específico, a la acción humana como núcleo educativo. Y así, como la misma persona se revela en la acción, el perfeccionamiento de la persona se obtiene en la educación a través de acciones.

De tal manera que, mientras la acción humana como tal, tiene originariamente una estructura conforme a la perfectibilidad de su naturaleza y por tanto es la finalidad de ésta, la acción educativa tiene una dimensión necesariamente intencional y, por lo mismo, de hecho, requiere de un adecuado fundamento teórico. Es sabido, pues, que la educación es concebida principalmente como proceso o como resultado, y en ambos casos, hace referencia a una perspectiva dinámica del ser humano. De tal modo, que será la Antropología Filosófica la que se convertirá en la vía correcta que permita la debida comprensión de la acción educativa, y así mismo de una adecuada Antropología Educativa. Esta última se puede, identificar (no exclusivamente) en su perspectiva sistemática con la Educación Personalizada, que en la segunda mitad de siglo ha sido acuñada por V. García Hoz a través de las notas educativas de: singularidad, autonomía y apertura, ${ }^{14}$ y últimamente ha quedado perfilada por el mismo autor, a través de un Sistema Fundamental de Objetivos de la Educación (S.O.F.E.) ${ }^{15}$, que abarca conocimientos, aptitudes y valores, y que, a su vez, se prolonga en el sistema de "Obra Bien Hecha"16, que apunta hacia los frutos del esfuerzo educativo. Cabe destacar, que en la presente investigación y desde el estudio de la dinámica personal, se aprecia como conveniente tener en cuenta respectivamente correlacionadas la educación personalizada en cuanto tal, en su misma eficiencia, y la "Educación Personalizada" según su vertiente sistemati- 
zadora en orden al planteamiento de su más específico autor, D. Víctor García Hoz.

Sin duda, el estudio de la Antropología Educativa realizado en cohesión con la Antropología Filosófica, permite clarificar y enriquecer el conocimiento sobre el ser humano. Y la posibilidad de plantear en estos tiempos una educación más auténtica, en tanto que personalizada. Por consiguiente, en la profundidad de este análisis se hace más patente el descubrimiento y reconocimiento radical de la persona como principio del proceso educativo, teniendo en cuenta que "el proceso educativo no se realiza ucrónica ni utópicamente"17. Surge así, según esta investigación naturalmente la constatación de la Educación Personalizada como precisión de la Antropología Educativa, como principio educativo y como criterio último en el cual se debe insertar y constatar cualquier planteamiento, aspecto y quehacer educativo referido a la educación, tanto en su vertiente natural como en la propiamente sistemática.

\subsection{Las dimensiones de la acción humana theoria, praxis y poiesis, como claves para la comprensión, aplicación y producción educativas}

Nuestro siglo, tan dado al tecnicismo ${ }^{18}$ y en sus interpretaciones sobre la realidad humana tan inclinado a diversos reduccionismos, que son analizados por un conocido autor contemporáneo como V. Frankl bajo el enjuiciamiento de que "el reduccionismo es el nihilismo de hoy"19. Por lo mismo, después de las indagaciones fragmentarias y analíticas, queda de relieve la conveniencia de poder afrontar una comprensión de conjunto, es decir, unificada en cuanto a los aspectos del ser humano. Por 
consiguiente, cabe sintetizar al ser humano en sus aspectos constructivos de la personalidad, según una referencia: biopsicosocial, y en cuanto a sus facultades: inteligencia y voluntad.

Así, evitando todo énfasis inadecuado, es conveniente indagar y presentar la más auténtica dinámica de reciprocidad en las facultades primordiales de acuerdo a su naturaleza en libertad. Pero justamente, ocuparse de la actividad humana implica advertir la gran facilidad con que se puede incurrir en desvirtuar la naturaleza de la misma, no sólo, según sea el caso, desde un enfoque desarticulado sino también desde una perspectiva indiferenciada. No se puede menospreciar el hecho de descubrir que la falta de conocimiento y dominio de sí mismo de la que ya se ha hecho mención ocurre de un modo peculiar; pues "no proviene de la dificultad de procurarnos la información necesaria, de su inexactitud o de su escasez. Al contrario, se debe, en no pocos casos, a la extremada abundancia y a la confusión de datos acumulados"20.

Sin duda, desde tantas posturas pseudorreales respecto de la acción humana, se resta autenticidad a la comprensión y ejecución de la acción educativa. Por ello, vale la pena ahondar en los grandes modos de obrar del ser humano, los mismos que pueden ser mejorados hasta conseguir su máxima efectividad. Así pues, las emergencias activas se distinguen en "theoria", "praxis" y "poiesis" como dimensiones que proceden de las facultades humanas susceptibles de ser diferenciadas y asimismo entrelazadas.

La multidimensión del ser humano, plasmada en: saber (teoría), obras (práctica) y produción (técnica), se corresponden con la pluralidad de las facultades humanas. Así la preeminencia la tiene la teoría, en razón de que "por naturaleza todo hombre desea saber" 21 Y 
esta dimensión ha de ser puntualizada con el hecho de que la teoría indica adhesión a la realidad "intención de conocer", aproximarse y poseer la verdad. De hecho, el hombre necesita darse razones teóricas, abstractas y universales e incluso necesita "saber saber". No corresponde, pues, al hombre obrar ciegamente y sí, ni la práctica, ni la técnica, son conocimientos en sí mismos y en esa medida, necesitan previamente de algún conocimiento, es decir, de la teoría, ésta debe de ir pues, siempre por delante.

Si se observa la acción de la especulación, resulta ser la condición propia del discurrir teórico; el mismo que avanza, elevándose hacia la sabiduría hasta lograr el nivel de contemplación. Por todo, se podría precisar que la sabiduría hunde sus raíces en la metafísica, la misma que permite entender al inteligible perfectísimo que es Dios, desde la operación más perfecta que es la de entender según nos enseña el Aquinate cuando precisa en su Suma Teológica que "la óptima operación del hombre es de la óptima potencia respecto del óptimo objeto"22. En este sentido: "la esencia de la felicidad consiste en un acto del entendimiento"23.

En realidad, ni la felicidad ni la contemplación son aditamentos, ni siquiera complementos, sino parte de la misma naturaleza humana. Cabría observar que "la tendencia del intelecto hacia la verdad, que es su fin, es la de una naturaleza, no la de un apetito" 24 . En fin, queda tanto por señalar acerca del quehacer y perfeccionamiento de la dimensión teorética. Pero conviene insistir en que el principio de todo acto humano está en el conocer; por lo mismo, toda acción educativa debe de acoger en su debida proporción, la exhortación: “¡Volvamos lo más pronto posible a los educadores que saben pensar antes que obrar!"25. 
Obviamente, si bien la vida humana debe estar regulada por la inteligencia, no se agota en esa facultad, ni en la dimensión cognoscitiva, sino que se abre a la acción. Al respecto hay que tener en cuenta que "así como la vida propia y esencial de la inteligencia es la contemplación, así la vida propia de la voluntad es la acción, la práctica"26.

Por tanto, nótese que hay en el hombre un sólo entendimiento, aunque a veces se lo diferencie según sus dimensiones y en orden a su uso y sobre todo según sus fines, capaz de funciones distintas. Así, desde la función teorética, el entendimiento versa sobre lo que es universal y necesario; y en orden a la función práctica de la razón, ésta se ocupa más bien, del "hic et nunc", de lo contingente y de la concreción particular. De tal modo, que la posibilidad del conocimiento práctico, es real, sólo como un conocimiento participado y derivado, que exige por su parte, la presencia de la teoría y la efectividad de la praxis. El pedagogo Comenio proponía, por lo mismo, que se debería enseñar: "A hablar hablando, a escribir escribiendo, a razonar razonando" 27 .

En consecuencia, con la especulación (theoria), se busca saber por saber; mientras, que con la acción (praxis) se pretende el ejercicio en orden a la misma acción y adecuada aplicación. Por tanto: "el saber práctico" tiene que ser un saber sobre la acción y conquistado en la misma acción; por eso su marco propio se descubre en el ámbito de la voluntad. Mas aún, la misma razón y raíz de la practicidad de la inteligencia reside en la voluntad (...). Sin duda, es en orden al fin y a la actividad volitiva que la inteligencia se ocupa de buscarlo, mejor aún, de señalar los medios, necesarios para su consecución" 28 .

Así pues, si por su dimensión teórica el hombre necesita saber, por su dimensión práctica el hombre necesita obrar y desde esta última, tendrá respecto de sí mis- 
mo que constituirse. De esta manera se convierte para sí en una tarea; cuyo quehacer posterior y paralelo se constituye en el proceso educativo. Fundándose y aspirando a la "recta ratio agibilium" (moral) y a la "recta ratio factibilium" (arte, técnica). Recuérdese que el entendimiento práctico "difiere del especulativo por el fin", como dice el "Doctor Communis": "En efecto, el fin del entendimiento práctico es la operación y el fin del entendimiento contemplativo es la posesión de la verdad; y por esto, cuando un arquitecto, por ejemplo, piensa como se puede construir un edificio, pero no con objeto de construirlo, sino primeramente, con el deseo de saber cómo se construye, quiere, con respecto al fin de su obra, obtener un conocimiento especulativo, aunque se trate de una cosa factible. Por consiguiente, la ciencia, que es especulativa, por parte de las cosas que conoce, es exclusivamente especulativa. La ciencia especulativa por el modo de conocer o por el fin que se propone, en parte es especulativa y en parte práctica, y aquella cuyo fin es la operación, es simplemente práctica"29.

\subsection{La interrelación entre la acción humana y la acción educativa}

Conviene tener presente la intrínseca relación natural que tendrá que devenir en intencional entre el orden del conocimiento práctico, la naturaleza humana y la acción educativa. Teniendo en mente que la regulación del obrar humano está inmersa en la consecución del "bien", en razón de su carácter de "fin". Ciertamente, la acción y la praxis tienden a un fin, a un objetivo determinante, sin

el cual pierden su dirección y su vitalidad; "la vida existe así mismo para un fin que la haga digna de ser vivida" 30 . De esta forma, en torno al orden (regulación), se nota la 
necesidad de la inteligencia por parte de la voluntad. Pero a su vez, el apetito racional moviliza a la misma razón empujándola. En cuanto a la vida humana, ésta es "praxis", por eso mismo, continuamente hay que realizarla; es imposible determinarla de una vez para siempre. En esta practicidad inmersa y propulsada por cambios, enseñanzas, aprendizajes, interviene la educación, y en esa medida se realiza el ser humano.

Se ha hablado de una "praxis" en cuanto que la acción y el fin permanecen en el agente; esta acción procura, pues, la perfección moral del ser humano. Pero hay otra "praxis" que es productiva, la "poiesis", y que en la medida que alude a la perfección de artefactos, de acuerdo a su ejecución transitiva, se diversifica de la primera, sin una separación radical, pero con una distinción esencial. Ha de precisarse así, que la acción productiva tiene su fin en aquello que produce. Por lo mismo, "tampoco se incluyen la una en la otra; en efecto, ni la acción es producción, ni la producción es acción"31.

Ahora bien, descubriendo la técnica y el arte en su perspectiva de habilidad, cabe analizar al filo del discurrir aristotélico que "puesto que la construcción es una técnica y es precisamente una disposición racional para la producción, y no hay técnica alguna que no sea disposición racional para la producción... cuyo principio está en el que lo produce y no en lo producido" 32 . Y en la medida que "toda técnica versa sobre el llegar a ser, y sobre el idear y considerar como puede producirse o llegar a ser algo... producción y acción son cosas distintas; la técnica o arte, tiene que referirse a la producción, no a la acción" 33 . Así, la "poiesis" se separan pues, la acción y el fin, como ya antes se ha mencionado. Por tanto, este rubro de los artefactos, las técnicas o los métodos, (en cuanto que, busca un efecto exterior), poseerá un carácter instrumental, y en esa medida tendrán una im- 
portancia definitivamente secundaria ${ }^{34}$, dentro del obrar humano inteligente.

Cuan necesaria es en esta época, la claridad sobre el proceder inteligente en sus distintas modalidades, para así acceder mejor al reconocimiento de una fe inteligente o de una inteligente fe, en razón de como diría San Agustín que, de tal manera, es por la fe, como se hace accesible, lo que de suyo es inaccesible; por ejemplo Cristo como Mediador. Vale pues la pena, aplicarse en el consejo de aquel gran escolástico y difusor de la obra Agustiniana, San Anselmo, Arzobispo de Canterbury, quien muy a principios del siglo XII exhortaba con insistencia: "fides quarens intellectum" 35 . De modo que, "Dios mismo, pasando por el Mediador, en la Persona de Cristo, por su secreta misericordia revelaste a los humildes y lo enviaste para que con su ejemplo aprendiesen hasta la misma humildad; aquel Mediador entre Dios y los hombres, tiene el nombre de Cristo Jesús y apareció entre los pecadores mortales Justo Inmortal, con los hombres, justo con Dios, para que el estipendio de la justicia unida a Dios fuese destruida en los impíos justificados en la muerte, que se dignó tener común con ellos... Porque en tanto es Mediador en cuanto Hombre, pues en cuanto Verbo no puede ser intermediario por ser igual a Dios, Dios en Dios y juntamente con él un solo Dios"36.

Sobre esta misma realidad trabaja Santo Tomás lo que por ejemplo de modo amplio, se puede observar en el tratamiento, dado a la estructura de la Suma Teológica: "Así pues, como quiera que el objetivo principal de esta Doctrina Sagrada (está haciendo referencia a la Teología), para llegar al conocimiento de Dios, y no sólo como ser, sino también como principio y fin de las cosas, especialmente de las criaturas racionales según ha quedado demostrado (c. 1, a. 7), en nuestro intento de exponer dicha doctrina trataremos de lo siguiente: primero, de Dios; se- 
gundo, de la marcha del hombre hacia Dios; tercero de Cristo, el cual, como hombre, es el Camino en nuestra marcha hacia Dios" ${ }^{37}$. Por lo demás, cabe también meditar, que Santo Tomás va a otorgar la debida profundidad a cada una de estas realidades al ocuparse de tales cuestiones de modo puntual.

\subsection{El cristianismo: orientación para la plenitud y la felicidad}

Indudablemente, la posibilidad total de ser sabio, y estar feliz en el amor, sólo será definitiva actualidad, en el encuentro del alma como lo más específico del hombre con Dios: Quien justamente, es capaz de dar cuenta de todo en cuanto Suma Verdad y colmar toda ansia de amor en razón de ser Suma Bondad. El filósofo que tiene por oficio ser sabio busca la causa primera: Dios. Y, con la escueta razón natural, es cierto, que la puede hallar; pero, si se le añade la Revelación, entonces, el descubrimiento es el de una Filosofía Cristiana y por ende la visión y el mismo encaminarse, es más completo. Además, en la línea de la Perfección, sólo puede haber, un único: "Ipsum Esse Subsistens"38. Por tanto, se trata de modo pleno de "percatarse" y "quedarse entusiasmado" o mejor dicho prendado del Principio y del Fin. Es que ipuede haber otra fuente tan digna para una total contemplación entregada?.

Por lo demás, los grandes santos han tenido muy en cuenta como también los insignes teólogos, que la Virgen es, a su vez, "Mediadora de todas las gracias" y modelo pleno de humanidad, se podría decir, que dentro de la misma alusión a la mediación en el proceso de graduación de perfeccionamiento humano, es decir en el itinerario de hacerse persona. La Virgen María entre los seres escuetamente pertenecientes a la especie humana; ocupa un 
lugar preferente dentro de la sabiduría alcanzada por los hombres, ciertamente, dada por el mismo Dios, pero refrendada por el quehacer de su personalidad. Por todo lo cual, los estudiosos de la Historia de la Filosofía si practican la honestidad intelectual, encontrarán en María, el emblema de la Filosofía Cristiana. Por su modo de discernir (theoria), y de decidir (praxis), en que se nota, tiene verdaderamente presente a la revelación divina, recogida en la Sagrada Escritura.

Para el caso, conviene hacer referencia al hecho que dentro de la investigación de este trabajo, y en plena reflexión del mismo, un día al acudir a la Santa Misa, en la época de la novena de la Inmaculada Concepción, escuché la Homilía de un sacerdote, cuyas palabras me hicieron pensar en la síntesis de la mediación de María, no sólo "iuxta crucem" como yo venía meditando, sino en general, desde la perspectiva de la Virgen como Medianera; para lo cual, se puede profundizar en aquel definitivo pasaje del milagro de la Boda de Caná en que la Madre de Dios y por eso, Madre de los hombres (pues en cuanto Madre de Cristo, lo es de toda la especie humana). Ciertamente, surge junto con Ella por su docilidad a la obra del Espíritu Santo, la posibilidad de comunicación con la naturaleza divina. Por lo mismo, se le oirá a la Madre de Dios, como Madre y Maestra, un consejo óptimo, en lo que se refiere al ejercicio de la libertad humana, para que ocurra el primer milagro: "Haced lo que él os diga" 39 transmitiendo Ella la sabia convicción, de que solo Cristo podía obrar como guía total y forjador íntimo frente a la posibilidad de aplicarse u obtener cualquier bien, con mayor razón, frente al hecho de lograr la plenitud de la personalidad humana y poder por último, poseer el Bien Sumo, y así la felicidad humana.

Para mayor explicación, destáquese también lo natural de todo acontecimiento sobrenatural. Por lo mis- 
mo, cabe percatarse, que en la redacción del Evangelio, es San Juan quien cuenta muy nítidamente, los acontecimientos antes mencionados. Nótese pues, que es el evangelista predilecto de Jesucristo quien recibió a la Virgen como Madre en aquel momento histórico de la Cruz cuando "Jesús, viendo a su madre y al discípulo a quien amaba, que estaba allí, dijo a su madre: Mujer he ahí a tu hijo. Después dice al discípulo: he ahí a tu madre. Y desde aquel momento, el discípulo la recibió en su casa ${ }^{40}$.

Todos estos hechos ponen de relieve, la permanente sintonía popular con la Madre por excelencia, que conduce a llamar a María "más que tú sólo Dios" y considerar como un proceder idóneo, "el ir a Jesús por María" más explícitamente, habría que considerar: "Omnes cum Petro ad Iesum per Mariam"; pues Ella es la que ha estado, y está, más íntimamente cerca de Cristo. Puesto que el hombre según ya se ha ido meditando en este trabajo, requiere siempre para ser él mismo, curiosamente, de medios, de ayuda, de imagen, de ejemplo. Para proceder con acierto en su propio "discurso de discernimiento" en el caso del saber y con una correcta "decisión de elección" frente al hecho concreto de obrar. Según hemos dicho, en razón a la llamada última de plenitud que se relaciona con cualquier acto que por último está impregnado de amor.

En tal sentido, "La Virgen María, Madre e imagen perfecta de la Iglesia, desde los comienzos del Nuevo Testamento ha sido proclamada bienaventurada, debido a su adhesión inmediata a la fe, sin vacilaciones frente a la Palabra de Dios (Cfr. Lc. 1, 38 45), que conservaba y meditaba permanentemente en su corazón (Cfr. Lc.2, 19 51). Ella se ha convertido así en modelo y apoyo para todo el Pueblo de Dios confiado a su cuidado maternal... nos muestra el camino de la acogida y del servicio a la Palabra, y al mismo tiempo, el fin último que jamás debe perderse 
de vista: el anuncio a todos los hombres y la realización de la salvación traída al mundo por su Hijo Jesucristo".41

Dirá Santo Tomás de Aquino: "En resumen, la Virgen María, es llena de gracia tanto por lo que mira a la práctica del bien, como por lo que toca a la evitación del mal". Por ello concluirá esta meditación en contemplación amorosa de constatación: "Bendita, pues, la Virgen; pero más bendito aún el fruto de su vientre:42.

Después de lo expuesto, cabe aún más precisar, que hay una analogía entre la vida de orden natural y la vida sobrenatural; y siendo esta última, superior y concluyente, permite realmente comprender y perfeccionar mejor en su integridad, la vida humana. A la vez, vale la pena, entrelazar y distinguir frente a ambos niveles el corolario de la sabiduría que en ambos casos se situará, en Dios. Por lo mismo, cabe descubrir, el imperativo délfico como una potencialidad inscrita en la naturaleza del hombre, mientras ésta queda tan enfáticamente recogida por Sócrates en el diálogo Platónico de Cármides o de la Sabiduría, cuya exhortación, se ve sintetizada en el lema: "Conócete a ti mismo y sé sabio"; aplicación, que de suyo, impulsa a la indagación y al descubrimiento de que: "La sabiduría es sin duda un gran bien; y si tú la posees eres un mortal dichoso... considérate por tanto dichoso cuanto más sabio seas" 43 .

Sobre esta base, se cultivan y expanden los conocimientos realistas. Así, más tarde, cuando se encuentra Aristóteles alejado de todo idealismo y pendiente de la realidad en sí, se manifiesta muy contento por el descubrimiento socrático de la definición, que lo induce a la aceptación de las cosas como son, a la captación de las esencias, después de notar inicialmente al ente. Por tanto, corresponde saber hacer frente a cualquier cosa o asunto, en cuanto ¿qué es? y asimismo ¿cómo es? y frente a todo ello, 
asombrarse, y dar cauce... como procede con el trabajo intelectual del ser humano. Más aún, en tal sentido, puede el hombre hacerse cargo, ¿qué es? y ¿por qué es? Por lo mismo, capaz de descubrir "que es capaz de conseguir", respuestas radicales, profundas, ordenables, demostrables... frente al bien deseado en cuanto ser humano.

Dentro de este proceder realista, se instaló Aristóteles en términos de quien indaga y busca aquello que es lo absoluto, digno de constituirse en fin y de propiciar una total unión frente al bien deseado por la naturaleza humana. El Estagirita, estudia por tanto, la dinámica de la vida humana, en razón del fin natural que tiene la misma. En tal sentido, en la obra titulada: "Ética a Nicómaco" en que se ocupa de la conducta del hombre expone: "todo arte y toda investigación, y del mismo modo toda acción y elección parecen tender a algún bien"44. Previamente, en la Metafísica del mismo autor, se recogen explicaciones en que bien, fin y acto se identifican entre sí.

Sin duda, si se procede con realismo al pensar y al obrar, se descubre la conexión enriquecedora, de abarcar los conocimientos de la vida natural, en tensión y apertura a la vida sobrenatural. Así, como ya se anotó más adelante, es imposible amar lo que no se conoce,... cómo ponerse en marcha hacia el fin sin más... Cuando, "el fin es, ciertamente, el último en el orden de ejecución, más el primero en la intención del agente, y en este sentido tiene condición de causa" ${ }^{45}$. Por tanto, si no se conoce con alguna claridad al absoluto, Dios; entendido como causa última para el conocer y el obrar; cómo se le puede amar. Pero, cómo conocerle acabadamente con las solas fuerzas humanas. No obstante, cuán valiosas son las posibilidades de la razón natural que llegan a explicaciones rigurosas sobre lo que pueden ser las referencias últimas para todo discurrir y obrar humano, en cuanto, 
las vías para aperturarse a la consecución del fin último sobrenatural.

Con tal bosquejo del fin humano, queda centrada la posibilidad sobrenatural desde la dinámica humana, "según noticia" como gusta hablar San Agustín en el Libro XIII de las "Confesiones": "Quisiera yo que conociesen los hombres en sí estas tres cosas. Cosas muy diferentes son estas tres de aquella Trinidad, más las menciono, para que las ejerciten en sí mismos y prueben y sientan cuán diferentes son. Y las tres cosas que digo son: Ser, conocer y querer. Porque yo soy, y, conozco y quiero: soy esciente y volente y se que soy y quiero ser y conocer. Véase, por tanto, quien pueda, en estas tres cosas, cuán inseparable resulta la vida siendo una la vida, y una la mente, y una la esencia, y cuán, curiosamente, inseparable de ella la distinción" 46 .

Se pone así de manifiesto, la conciencia de insuficiencia por parte del hombre, frente al orden sobrenatural. No obstante, la necesidad de trabajar interiormente, primero, abriéndose a su propio mundo natural, y "desde sí, estar dispuesto en oración:... Ciertamente que cada uno está delante de sí; así que atienda a su interior y vea y hábleme después"... ${ }^{47}$. "Gracias a Dios, tener "noticia" de su Palabra en sentido más propio: Cristo que como real fundamento respondió de sí: "Yo soy el Camino, la Verdad y la Vida; nadie va al Padre sino por mí"48. Y así, en razón de la naturaleza humana, se requiere de culminar ésta, en la divina; y desenvolverse por ejemplo, desde el reconocimiento que se puede apreciar, en la obra "Confesiones" de San Agustín, cuando dice en el primer punto del primer capítulo del primer libro: “...nos has hecho para Tí y nuestro corazón está inquieto hasta que descanse en Tí”. 


\section{Notas:}

1 Aquino, Tomás de, Sto. Suma Teológica, I, c. 29, a 3.

2 Ibídem, De Potentia, 9. a 3.

3 Agustín de Hipona, San, Soliloquios, Lib. I, cap. II.

4 Aristóteles, Acerca del Alma. Lib. I, cap. II, 403 b 25.

5 Ibídem, 404 b 5.

6 Ibídem, Lib. II, cap. IV, 412b 5.

7 Aristóteles, Acerca del alma. Lib. II, cap. IV, $412 \mathrm{~b} 15$.

8 Agustín de Hipona, San, Confesiones, Lib. X, cap. VI, 11, B.A.C., Madrid, 1946, pp. 717719.

9 Millán Puellés, A., Persona humana y justicia social, 1982 (a), p. 13.

10 Millán Puellés, A., Persona humana y justicia social, 1982, pp. 1213.

11 Wojtyla, K., Persona y acción, 1982, p. 13.

12 Aristóteles, Física, Lib. II, cap. I.

13 Aristóteles, Ética a Nicómaco. Lib. I, cap. IV.

14 García Hoz, V. Educación Personalizada, Rialp, Madrid, 1985

15 Ibidem, Práctica de la educación personalizada, 1988, págs. 47147.

16 Ibidem, págs. 157278.

17 Ibañez Martín, J.A..., Hacia una formación humanística, 1982, pág. 63.

18 Maritain, J. La educación en este momento crucial, 1985 págs. 1135.

19 Frankl, V., La voluntad de sentido, 1988. pág. 18.

20 Carrel, A., La incógnita del hombre, 1946, p. 51.

21 Aristóteles, Metafísica, Lib. I, cap. 1.

22 Aquino Tomás de, Sto., Suma Teológica, III, c.3, a.5.

23 Ibidem, III, c.3, a.4.

24 Gilson, E., El amor a la sabiduría, 1974, p. 53

25 Morín, L., Los charlatanes de la nueva pedagogía, 1975, p. 233

26 Derisi, O.N., La doctrina de la inteligencia de Aristóteles a Santo Tomás, 1981 , p. 218

27 Comenio, A., Didáctica Magna, cap. XXI

28 Derisi, O.N., La doctrina de la inteligencia de Aristóteles a Santo Tomás, 1981, pág. 218.

29 Aquino, Tomás de, Sto. Suma Teológica, I, c.14, a. 16.

30 Maritain, J., La educación en este momento crucial, 1981 pág. 23.

31 Aristóteles, Ética a Nicómaco, Lib. VI, cap. 1, 1140 a.

32 Aristóteles, Ética a Nicómaco, Lib. VI, cap. 1, 1140 a.

33 Aristóteles, Ética a Nicómaco, Lib. VI, cap. 1, 1140 a. 
34 Millán Puellés, A., "El sentido trascendente de la existencia y de la educación”. En Rev. Española de Pedagogía, n.158, 1982, pág. 158. a la que está llamado. De allí, que es la mediación para alcanzar el fin último, sobrenatural, que no puede ser otro.

35 Anselmo de Canterbury, San, Proslogium, cap. 1.

36 Agustín de Hipona, San, Confesiones, Lib. X, cap. 43, 68, B.A.C., Madrid, 1946, pág. 783.

37 Aquino, Tomás de, Sto., Suma Teológica, c.2, Prólogo.

38 Ocariz, F., "Rasgos fundamentales del pensamiento de Santo Tomás" págs. 5089. En, Las razones del Tomismo. Fabro, C., Ocariz, F., y otros. EUNSA, Pamplona, 1982, págs. 064.

39 Jn., cap. 2, ver. 5.

40 Jn., cap. 19, ver. 27.

41 Ratzinger, J. Card., Instrucción sobre la vocación eclesial del teólogo, Roma, 24 de Mayo de 1990, Conclusión.

42 Aquino, Tomás de, Sto. Exposición sobre el Ave María. págs. 181187. En, Escritos de Catequesis, Rialp, Madrid, 1978, pág. 187.

43 Platón, "Cármides" o "De la Sabiduría". En, Obras Completas, Aguilar, Madrid, 1966.

44 Aristóteles, Ética a Nicómaco, Lib. I., cap. I, 1, 1094 a.

45 Aquino, Tomás de, Sto., Suma Teológica, 12, c.1, a.1, 1.

46 Agustín de Hipona, San, Confesiones, Lib. XIII, cap. 11, 12., B.A.C., Madrid, 1946, pág. 915.

47 Agustín de Hipona, San, Confesiones, Lib. XIII, cap. 11, 12., B.A.C., Madrid, 1946, p. 915.

48 Jn., cap. 14, ver. 6.

\section{Bibliografía citada}

\section{AGUSTÍN DE HIPONA, SAN,}

1946 "Los Soliloquios" Vol. I.; "Confesiones" Vol II. En Obras Completas, B.A.C., Madrid.

ANSELMO DE CANTERBURY, SAN,

1970 "Proslogion", Aguilar, Madrid.

AQUINO TOMAS DE, SANTO,

1949 Quaestio Disputata de Potentia. Spiazzi, Marietti; Taurini, Roma.

1978 "Exposición sobre el Ave María". En, Escritos de Catequesis, Rialp, Madrid. 


\section{ARISTOTELES,}

1983 Acerca del Alma. Gredos, Madrid.

1985 Etica a Nicómaco. Centro de Estudios Constitucionales. Madrid.

BOECIO, A.M.T.S.,

1973 La consolación de la filosofía. Aguilar, Madrid.

CARREL, A.,

1946 La incógnita del hombre. A. Joaquín Gil, Buenos Aires.

COMENIO, J.A.,

1972 Didáctica Magna. Reus, Madrid.

DERISI, O.N.,

1981 La doctrina de la inteligencia de Aristóteles a Santo Tomás. Club de Lectores, Buenos Aires.

1979 Concepto de la Filosofía Cristiana. Club de Lectores, Buenos Aires.

ELDERS, L. S.V.D.,

1991 “Les Theories de L'Historicité de la Pensée et S. Thomas D’Aquin”. En, Atti del Congreso Tomístico Internazionale, Vol. I. Citta del Vaticano.

FABRO, C.,

1980 "Santo Tomás frente al desafío del pensamiento moderno". En, Las razones del Tomismo, Fabro, C. Ocariz, F., Vansteenkiste, C., Livi A., y otros. EUNSA, Madrid.

1960 La nozione metafísica di participazione, S.E.I., Torino.

FRANKL, V.E.,

1988 La voluntad de sentido. Herder, Barcelona.

GARCIA HOZ, V.,

1985 Educación Personalizada. Rialp, Madrid.

GARRIGOU LAGRANGE, R.,

1980 La existencia de Dios. Palabra, Madrid.

GILSON, E.,

1981 El Espíritu de la Filosofía Medieval. Rialp, Madrid.

1962 El Filósofo y la Teología. Guadarrama, Madrid. 